\title{
Counting forbidden patterns in irregularly sampled time series. II. Reliability in the presence of highly irregular sampling
}

Konstantinos Sakellariou, Michael McCullough, Thomas Stemler, and Michael Small

Citation: Chaos 26, 123104 (2016); doi: 10.1063/1.4970483

View online: http://dx.doi.org/10.1063/1.4970483

View Table of Contents: http://aip.scitation.org/toc/cha/26/12

Published by the American Institute of Physics

\section{Articles you may be interested in}

Counting forbidden patterns in irregularly sampled time series. I. The effects of under-sampling, random depletion, and timing jitter

Chaos: An Interdisciplinary Journal of Nonlinear Science 26, 123103 (2016); 10.1063/1.4968551

Local noise sensitivity: Insight into the noise effect on chaotic dynamics

Chaos: An Interdisciplinary Journal of Nonlinear Science 26, 123102 (2016); 10.1063/1.4970322

Method for reconstructing nonlinear modes with adaptive structure from multidimensional data

Chaos: An Interdisciplinary Journal of Nonlinear Science 26, 123101 (2016); 10.1063/1.4968852

Tangent map intermittency as an approximate analysis of intermittency in a high dimensional fully stochastic dynamical system: The Tangled Nature model

Chaos: An Interdisciplinary Journal of Nonlinear Science 26, 123105 (2016); 10.1063/1.4968207

On the limits of probabilistic forecasting in nonlinear times series analysis

Chaos: An Interdisciplinary Journal of Nonlinear Science 26, 123114 (2016); 10.1063/1.4971412

Classical investigation of long-range coherence in biological systems

Chaos: An Interdisciplinary Journal of Nonlinear Science 26, 123116 (2016); 10.1063/1.4971963

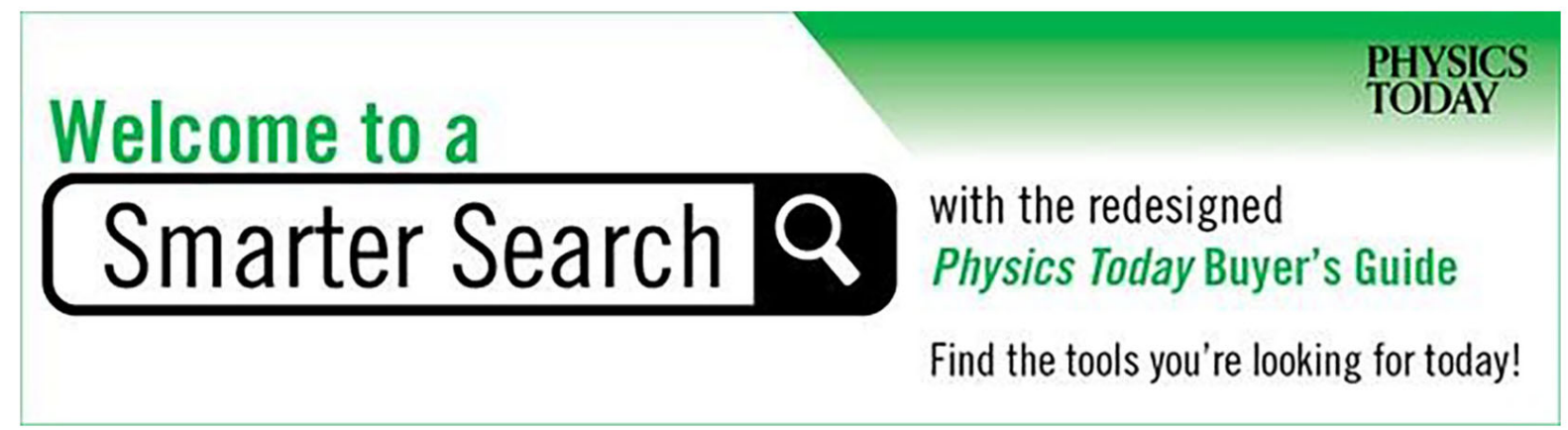




\title{
Counting forbidden patterns in irregularly sampled time series. II. Reliability in the presence of highly irregular sampling
}

\author{
Konstantinos Sakellariou, ${ }^{1, \text { a) }}$ Michael McCullough, ${ }^{1}$ Thomas Stemler, ${ }^{1,2}$ \\ and Michael Small ${ }^{1,2,3}$ \\ ${ }^{1}$ School of Mathematics and Statistics, University of Western Australia, 35 Stirling Hwy, \\ Crawley, WA 6009, Australia \\ ${ }^{2}$ Complex Data Modelling Group, Faculty of Engineering, Computing and Mathematics, \\ University of Western Australia, 35 Stirling Hwy, Crawley, WA 6009, Australia \\ ${ }^{3}$ Mineral Resources, CSIRO, Kensington, WA 6151, Australia
}

(Received 23 September 2016; accepted 14 November 2016; published online 5 December 2016)

\begin{abstract}
We are motivated by real-world data that exhibit severe sampling irregularities such as geological or paleoclimate measurements. Counting forbidden patterns has been shown to be a powerful tool towards the detection of determinism in noisy time series. They constitute a set of ordinal symbolic patterns that cannot be realised in time series generated by deterministic systems. The reliability of the estimator of the relative count of forbidden patterns from irregularly sampled data has been explored in two recent studies. In this paper, we explore highly irregular sampling frequency schemes. Using numerically generated data, we examine the reliability of the estimator when the sampling period has been drawn from exponential, Pareto and Gamma distributions of varying skewness. Our investigations demonstrate that some statistical properties of the sampling distribution are useful heuristics for assessing the estimator's reliability. We find that sampling in the presence of large chronological gaps can still yield relatively accurate estimates as long as the time series contains sufficiently many densely sampled areas. Furthermore, we show that the reliability of the estimator of forbidden patterns is poor when there is a high number of sampling intervals, which are larger than a typical correlation time of the underlying system. Published by AIP Publishing.
\end{abstract}

[http://dx.doi.org/10.1063/1.4970483]

Detecting a deterministic component in noisy data is an important problem in nonlinear time series analysis. The assumption of determinism underlies a large class of techniques that focus on the theory of dynamical systems. Symbolic dynamics tools have recently shown potential towards this goal. Ordinal patterns, in particular, comprise symbols obtained by a segmentation of a time series into elements of equal length. Patterns that cannot occur for a specified system are termed forbidden. Analysing the statistical properties of the resulting sequence can shed light on the underlying dynamics. Deterministic time series are thought to always be characterised by forbidden patterns, in contrast to random systems whereby any possible pattern may be realised. Thus, the relative proportion of forbidden patterns can be used to detect determinism. We investigate the effects of highly irregular sampling, such as from paleoclimate or geological data, on the reliability of this statistic as estimated from time series data.

\section{INTRODUCTION}

Real-world data sets often appear in the form of nonuniformly sampled time series. This may be due to device failure, weather conditions, human error, the nature of the system (e.g., financial transactions data) or the measurement method (e.g., geological data), and other causes. For

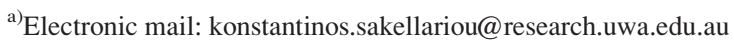

this study, we are motivated by geoscientific ${ }^{1}$ and paleoclimate time series, ${ }^{2-4}$ which are characterised by missing entries and large chronological gaps. Although there exist several types of irregular sampling, which can vary from rather mildly to highly unevenly spaced data, the majority of established techniques in time series analysis assume regular sampling. Consequently, there is an increasing need to extend the applicability of existing techniques and create more sophisticated tools to reliably analyse irregularly sampled time series. For linear systems, the Lomb-Scargle periodogram (also known as Vanicek's least-squares method $)^{5-7}$ is an example towards this direction. For nonlinear systems, there exist a few notable (but very recent) examples, such as the similarity estimators proposed by Rehfeld and Kurths, ${ }^{8}$ the distance metric for marked point processes by Suzuki et al., ${ }^{9}$ and the transformation-cost time series approach. ${ }^{10,11}$

Detecting a deterministic component in a given time series is an important pursuit within the field of nonlinear dynamics as this very assumption underlies all techniques that require a phase-space reconstruction. Analysing the symbolic dynamics of patterns extracted from a data set has displayed significant potential. A process by which a time series is mapped to a symbolic sequence comprising permutations of a finite set of natural numbers was first introduced by Bandt and Pompe. ${ }^{12}$ Ordinal patterns associated with a time series are obtained by partitioning the data set into elements of equal size. The symbol corresponding to each element reflects the rank ordering of sample points within. By 
recording the relative frequency of occurrence of each symbol, an empirical probability distribution is constructed, which contains partial information about the dynamics of the underlying system. Measures applied to this empirical distribution, for instance the original permutation entropy, ${ }^{12}$ can shed light on the system's complexity. Several studies that explore this methodological approach have been published since then. Its applicability to real-world systems was investigated by considering brain wave data, ${ }^{13,14}$ Electroencephalography (EEG) signals of epilepsy patients ${ }^{15}$ and heart variability interbeat data. ${ }^{16}$

While chaotic systems can closely mimic stochastic behaviour, the deterministic basis of chaos dictates certain constraints in the set of ordinal patterns, which can occur in a time series, ${ }^{17}$ hereinafter called admissible patterns. In other words, deterministic systems are characterised by the existence of a set of forbidden patterns-patterns that do not occur in any time series realisations thereof-whereas random systems can admit every possible ordinal pattern given sufficiently long time series. This stems from the fact that sampling a stochastic process will generate identically and independently distributed (i.i.d.) values. Mapping the sample data set to a symbolic alphabet of finite size (there are only $m$ ! total possible permutations of order $m$ ) results in a sequence of i.i.d. permutations, i.e., a stochastic process of finite discrete state space. The probability that any ordinal pattern is missing approaches zero as the total number of data points grows $(N \rightarrow \infty){ }^{18}$ The forbidden patterns' property was proved for piecewise monotone interval maps on an one-dimensional closed interval, ${ }^{19}$ but has also been empirically observed in data sampled from continuous systems. ${ }^{20,21}$

Kulp et $a l .{ }^{20}$ studied the reliability of the relative count of forbidden patterns as a signal of determinism in certain types of irregularly sampled data. In a previous study, ${ }^{21}$ we extended this investigation by exploring the parameter space of the method, the patterns' length, and highlighting the importance of choosing a value as large as possibly permitted by the length of the data set. We then conducted numerical experiments under this new scope on both regularly sampled data (of various sampling periods) and irregularly sampled data—resulting from (a) random depletion and (b) timing jitter. Results on the measure's robustness were interpreted from a nonlinear dynamics perspective.

In this paper, we further extend upon previous results by exploring cases of more "severe" irregular sampling, far removed from the regular sampling grid archetype. The types of unevenly sampled synthetic data we examine are more characteristic of geoscientific measurements, such as geological or paleoclimate data, and pose a formidable challenge for the ensuing time series analysis under any methodological approach. ${ }^{11}$ The question we ask is about the reliability of the estimator of forbidden patterns as a criterion for the existence of determinism in highly irregularly sampled time series. The sampling schemes (sketch in Fig. 1) we examine here include a Poisson sampling process (exponentially distributed intervals), Pareto sampling (example of a power-law distribution), and $\Gamma$ distributed sampling periods for various levels of skewness.

We briefly describe the concept of ordinal patterns in Section II and outline our methodology for estimating the

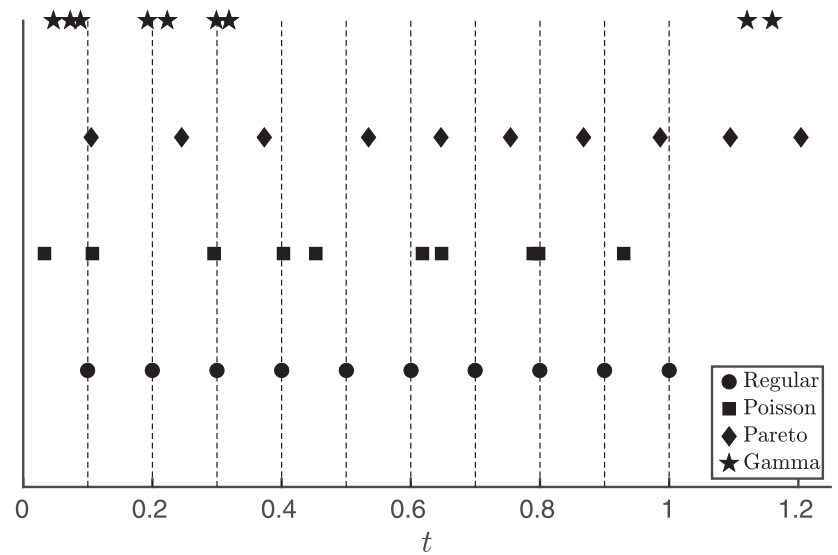

FIG. 1. Contrast between regular and irregular sampling schemes. Exponentially (Poisson sampling), Pareto and Gamma distributed sampling intervals of fixed mean $\Delta t=0.1$. Sketch of 10 successive sample points.

relative number of forbidden patterns in various irregularly sampled data in Section III. Our results are presented in Section IV, followed by a discussion of our findings and conclusions in Section V.

\section{ORDINAL PATTERNS}

Ordinal patterns ${ }^{12}$ are obtained by segmenting a time series into equal-length windows and considering the rank ordering of points within each segment. Let $\left\{x_{n}\right\}_{n=1}^{N}$ denote an arbitrary scalar time series of $N$ measurements ordered in a temporal fashion. Each segment of fixed size $m$ is denoted by the $m$-tuple $\mathbf{x}_{i}^{(m)}=\left(x_{i}, x_{i+1}, \ldots, x_{i+m-1}\right) \in \mathbb{R}^{m}$ for $i=1,2, . ., N-m+1$. The scheme is overlapping (successive segments have $m-1$ points in common), akin to sequentially sliding a window over the data by a lag of 1 point each step. If the time series is generated by a deterministic dynamical system, the segmentation into elements of size $m$ may be thought of as a partition of the underlying state space into $m$ ! regions.

Each of the $N-m+1$ elements is mapped to a symbolic ordering, a permutation of the natural numbers $\{1,2, \ldots, m\}$, depending on the relative amplitude of elements within the partition, in ascending order. Consider the case $m=5$, for instance, and a time-indexed example segment, say, the third chronologically in the time series, $\left(x_{3}, x_{4}, x_{5}, x_{6}, x_{7}\right)$, whereby $x_{5}<x_{7}<x_{4}<x_{3}<x_{6}$. First, we shift the time indices to the set $\{1,2,3,4,5\}$. Then, the associated permutation is $(3,5,2,1,4)$ because the 1 st point of this segment chronologically $\left(x_{3}\right)$ is ranked fourth, the 2nd $\left(x_{4}\right)$ is ranked third, the $3 \mathrm{rd}\left(x_{5}\right)$ is ranked first, the 4 th $\left(x_{6}\right)$ is ranked last, and the 5th $\left(x_{7}\right)$ is ranked second. In other words, the chronological indices within a segment are stored as elements in a vector indexed by the corresponding ranking.

In general, an ordinal pattern of order $m$

$$
\Pi_{i}^{(m)}=\Pi\left(\mathbf{x}_{i}^{(m)}\right)=\left(\pi_{1}, \pi_{2}, \ldots, \pi_{m}\right), \pi_{j} \in\{1, \ldots, m\}
$$

is defined as the transformation $\Pi: \mathbb{R}^{m} \rightarrow \mathcal{S}_{m}$ such that

$$
x_{i-1+\pi_{1}}<x_{i-1+\pi_{2}}<\ldots<x_{i-1+\pi_{m}},
$$


where $\mathcal{S}_{m}$ denotes the set over which the permutation group of order $m$ acts. In the event of two elements of $\mathbf{x}_{i}^{(m)}$ being equal, we pick the one that occurs first chronologically as the smallest. Since the cardinality of $\mathcal{S}_{m}$ is $m$ !, the relative number of forbidden patterns (over the set of all possible) associated with a specified time series is given by

$$
\mathcal{P}_{f}^{(m)}\left(x_{t}\right)=\frac{m !-\left|\bigcup_{i=1}^{N-m+1} \Pi_{i}^{(m)}\right|}{m !}
$$

i.e., the relative number of ordinal $m$-patterns that were not realised in this time series. The union $\cup \Pi_{i}^{(m)}$ in the above formula denotes the set of distinct ordinal $m$-patterns observed in a given time series (visible) and the symbol $|$. denotes cardinality. In practice, $\mathcal{P}_{f}^{(m)}\left(x_{t}\right)$ is an estimator for the relative count of forbidden patterns in the case of $N<\infty$. Note that the absence of an $m$-pattern pervades all longer patterns for larger $m$ in the form of outgrowth forbidden patterns, which leads to super-exponential growth in contrast to admissible $m$-patterns (exponential). This has been theoretically described for maps ${ }^{22}$ and numerically shown for the synthetic time series we consider here. ${ }^{21}$

\section{METHODOLOGY}

In order to examine the effects of sampling on the estimator of the relative proportion of forbidden patterns computed from a time series, we generated synthetic data for various irregular sampling schemes. To address the common practical issues associated with ordinal patterns, ${ }^{22}$ such as finiticity or noise, we simulated very long deterministic trajectories from the well-studied Lorenz system in the absence of noise. To avoid undersampling, the condition $N \gg m$ ! $+m-1$ needs to hold. ${ }^{22}$ For comparison purposes, we use the same parameter regimes as in Ref. 20 and our previous study $^{21}$ with $\rho=200, \beta=\frac{8}{3}$, and (a) $\sigma=100$ or (b) $\sigma=25$ for periodic and chaotic dynamics, respectively. Time series of 20000 time units were generated after transient effects were removed, which corresponds to approximately 89726 cycles in the periodic data (mean cycle period $T_{\text {mean }} \simeq 0.22$,
Fig. 2(a) and 64788 cycles in the chaotic data $\left(T_{\text {mean }} \simeq 0.31\right.$, Fig. 2(b)). We pick the $x$-coordinate for the ensuing numerical experiments. For additional verification of our findings, we repeated all experiments by computing ordinal patterns from $x$-component time series generated by the Rössler system in an 8-periodic regime $(a, b, c)=(0.3848,2,4)$ (Fig. 2(c)) and the four-dimensional (4D) Rössler system in a hyperchaotic regime $(a, b, c, d)=(0.25,3,0.5,0.05)$ (Fig. 2(d)), for similarly long trajectories. Different $\Delta t$ values to the Lorenz examples are used to ensure an approximate correspondence of the sampling frequency (rather than the period) among experiments with different data sets.

Consider a regular sampling grid with sampling intervals of fixed size $\Delta t$, and sampling times $\tau_{k}=k \Delta t, k=0,1,2, \ldots$. The corresponding sampling rate is constant and equal to $\Delta t^{-1}$ points per unit time. In order to sample in a highly irregular fashion, we consider three different distributions. Henceforth, sampling intervals $t_{k}=\tau_{k+1}-\tau_{k} \in \mathcal{T}_{k}$ are not constant, but rather independently and identically distributed random variables. We are interested in the three following cases. First, sampling $\tau_{k}$ by means of a Poisson process, in which case sampling intervals $\mathcal{T}_{k} \sim \operatorname{Exp}(\lambda=\Delta t)$. The sole parameter of the exponential distribution, the intensity $\lambda$, is set to the value of a characteristic sampling interval of a corresponding regular grid, $\Delta t$, so as to fix the mean $\mathrm{E}\left[t_{k}\right]$ as a reference for comparison. Second, we sample using intervals drawn from a power-law distribution, specifically $\mathcal{T}_{k} \sim \operatorname{Pareto}\left(x_{m}=\Delta t, \alpha\right)$, where the shape parameter $\alpha=10$ (tail index) is such in order to ensure a non-vanishing probability for only moderately sized "chronological gaps" (larger sampling intervals in comparison to a typical $\Delta t)$. The scale parameter $x_{m}$ is set to $\Delta t$ and, hence, $\mathrm{E}\left[t_{k}\right]=\frac{10 \Delta t}{9}$. Finally, we sample from a Gamma distribution of varying skewness where $\mathcal{T}_{k} \sim \Gamma\left(\alpha, \theta=\frac{\Delta t}{\alpha}\right)$. Its density function is defined by

$$
f_{\mathcal{T}_{k}}\left(t_{k} ; \alpha, \theta\right)=\mathrm{P}\left[\mathcal{T}_{k}=t_{k}\right]=\frac{T_{k}^{\alpha-1} e^{-\frac{t_{k}}{\theta}}}{\theta^{\alpha} \Gamma(\alpha)}, \quad t_{k}>0, \alpha, \theta>0,
$$

whereby the shape parameter $\alpha=\left(\frac{2}{\operatorname{Skew}\left(\mathcal{T}_{k}\right)}\right)^{2}$ depends only on the skewness of the distribution. The shape parameter
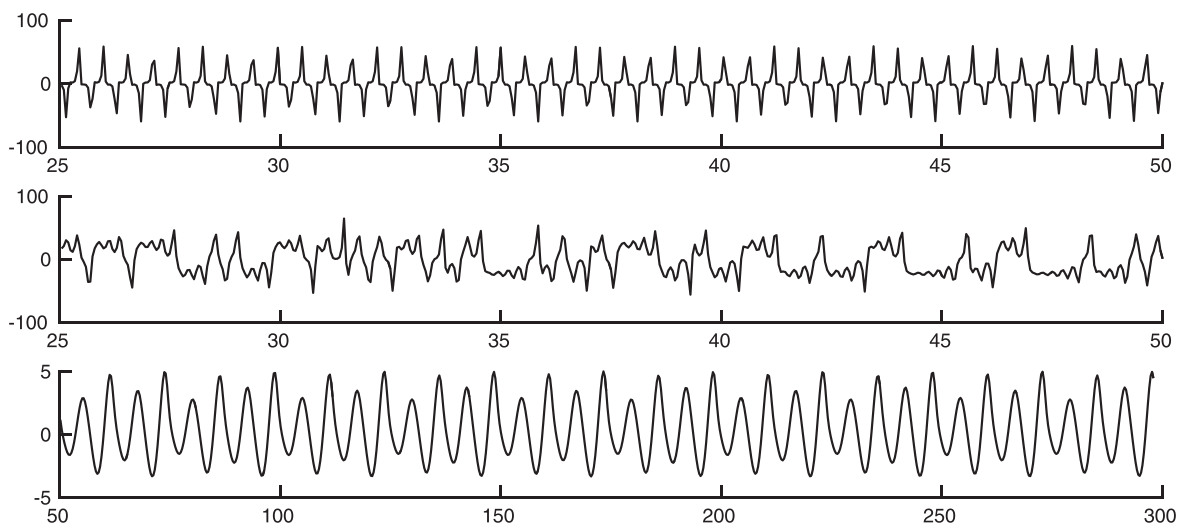

FIG. 2. Sample of (a) periodic Lorenz, (b) chaotic Lorenz, (c) 8-periodic Rössler, and (d) hyperchaotic 4D Rössler trajectories.

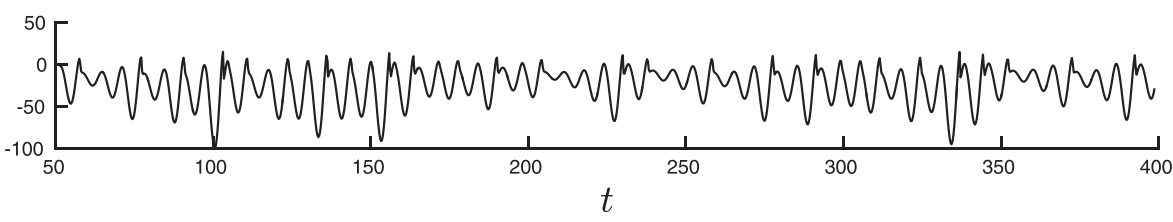


$\theta=\Delta t / \alpha$ is defined by means of the formula for the first moment $E\left[t_{k}\right]=\alpha \theta$, again fixed to $\Delta t$. A $\Gamma$-distribution with varying skewness can capture several types of irregular sampling, ranging from normal to exponential to power-law distributions. Empirical sampling distributions, from paleoclimate data in particular, ${ }^{3}$ seem to follow the $\Gamma$-paradigm with very high skewness (large gaps in between dense areas). When $\operatorname{Skew}\left(t_{k}\right)=2$, it is equivalent to an exponential distribution of intensity $\Delta t^{-1}$. As $\alpha \rightarrow \infty$ and the skewness decreases at a rate proportional to $\alpha^{-\frac{1}{2}}$, it approaches a normal distribution with $\mu=\Delta t$ and $\sigma^{2}=\frac{\Delta t^{2}}{\alpha}$. We vary the skewness in accordance with the analysis performed by Rehfeld et l. $^{3}$

\section{RESULTS}

To demonstrate how sampling by means of a Poisson process affects the estimated proportion of forbidden patterns in the periodic and chaotic Lorenz data, we plot the percentage of forbidden patterns as a function of the sampling intensity $\Delta t$ in Fig. 3 for $4 \leq m \leq 8$ and the corresponding experiments on regularly sampled data of period $\Delta t$ with $m=7$.

Recall that periodic systems are highly regular and generally admit only a very small number of $m$-patterns, smaller with increasing pattern length by virtue of the outgrowth property. Thus, the relative count of forbidden patterns is very large and $\mathcal{P}_{f}^{(m)}$ usually lies in the highest decile $(>90 \%)$
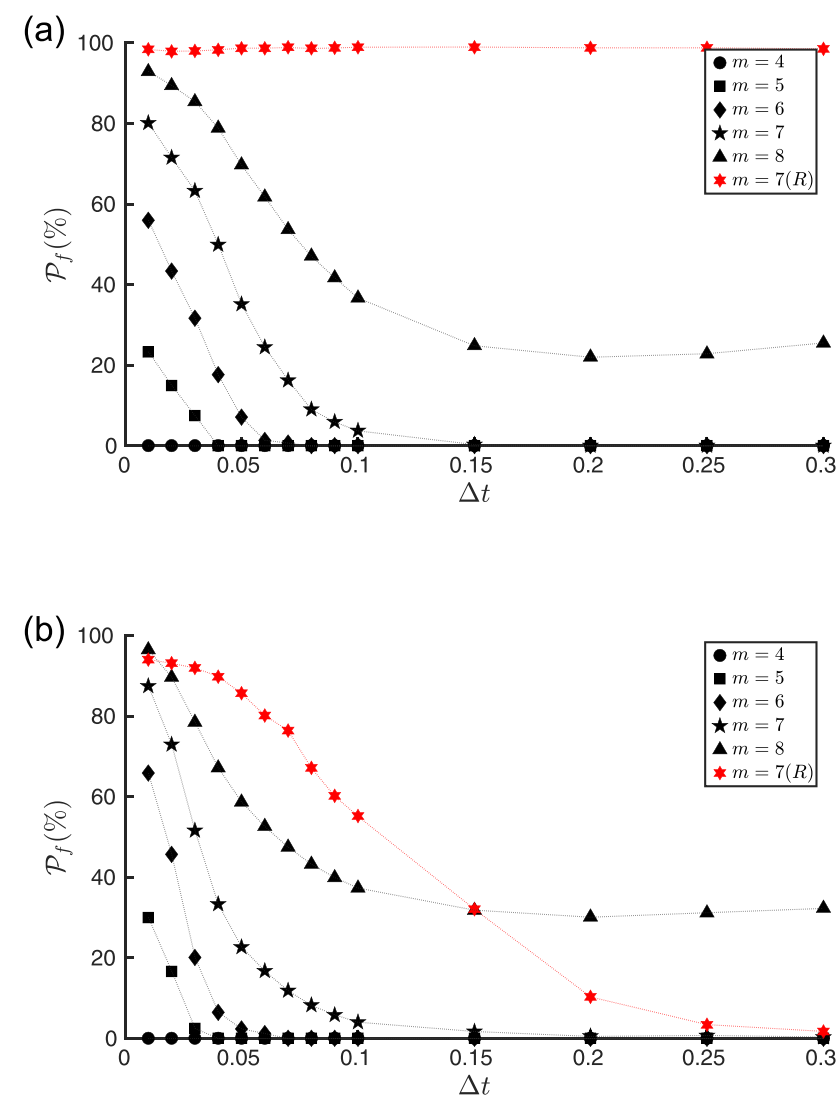

FIG. 3. Proportion of forbidden patterns in periodic (top panel) and chaotic (bottom) Lorenz time series sampled by means of a Poisson process for various intensities with mean sampling interval $\Delta t$ and $4 \leq m \leq 8$. The $m=7$ curve for regularly sampled data of fixed interval $\Delta t$ is shown in red. provided $m$ is not too small. This is also evidenced by the cross marks depicting the case $m=7$ of evenly spaced data (red) in Fig. 3(a). In comparison to regular sampling, there is a dramatic drop in $\mathcal{P}_{f}^{(m)}$ in the case of Poisson sampling for both periodic and chaotic time series (Fig. 3(b)).

Three key observations can be made from Fig. 3. First, $\mathcal{P}_{f}^{(m)}$ decays to zero very rapidly in the cases of small pattern length $m=4,5$. This implies that false admissible patterns are detected for almost all mean sampling intervals, patterns that should not be present (type I error in statistical terms, a "false positive"). The estimator is very unreliable if $m$ is small and can lead to misleading conclusions, failing to detect any deterministic component completely. Even with $m=6$ and a mean sampling interval of $\sim 0.05-0.06$ (17-20 points per unit time), almost no forbidden patterns are detected with Poisson sampling. This is in accordance with our previous conclusions about the unreliability of the $\mathcal{P}_{f}^{(m)}$ estimator when $m$ is small. ${ }^{21}$ The effect is even more pronounced in the case of Poisson sampling.

Second, if $m \geq 7$, there is a substantial increase in the count of forbidden patterns but only for smaller mean sampling intervals. There is a huge discrepancy in the periodic case between the two sampling schemes as $\Delta t$ increases. This can be seen by comparing $\mathcal{P}_{f}^{(7)}$ in Fig. 3(a) for regularly and Poisson sampled data when $\Delta t>0.1$. The estimator is detecting a particularly high number of forbidden patterns in the regular case, indicating a very dominant deterministic component (as expected), while none in the Poisson case for $m=7$ pointing to white noise. This is due to the fact that regularly sampled periodic data will always manifest their periodicity in the count of forbidden patterns irrespective of sampling period. In other words, the estimator will always detect regularity and periodicity even if aliasing due to undersampling leads to measuring a period greater than that of the original signal. ${ }^{21}$ When data are obtained by Poisson sampling, on the contrary, the estimator leads to increasingly more false admissible patterns as the mean sampling interval becomes larger and gradually decays to zero. Note that this is not the case when $m=8$ because at some point, around $\Delta t \simeq 0.25$, the effects of undersampling due to insufficiently long data sets (the condition $N \gg m !-m+1$ fails as $N \simeq$ 67000 and $8 !=40320)$ counterbalance the effects of the irregularities in the sampling interval. False forbidden patterns in the sense of Amigó et al. ${ }^{22}$ arise (Type II error, "false negative"), which make the estimator increase again with $\Delta t$, once higher than 0.25 approximately, and will eventually approach 1 .

Third, the chaotic case (Fig. 3(b)) indicates similar flaws in the detection of forbidden patterns, but the discrepancy compared to regular sampling is smaller if the mean sampling interval is small. In particular, comparing the two schemes for $m=7$ as previously, one can see that the count for $\Delta t=0.01$ differs only by approximately $5 \%$ or less. This is consistent with our previous findings on the relationship between the mean sampling period and the decorrelation and/or Lyapunov time of a chaotic system. ${ }^{21}$ Sampling irregularly severely affects the count of forbidden patterns; however, if the resolution is high enough, the estimator can be very reliable. Larger mean sampling intervals, which are 
closer to the system's decorrelation time, can lead to uncorrelated consecutive measurements. These measured values will in turn lead into the introduction of novel false admissible patterns, followed by a consequent drop of the forbidden patterns' estimator to zero. This implies that the mean sampling interval is a more significant factor when sampling chaotic systems.

Finally, note that comparison between the $m=7(R)$ curves of Figs. 3(a) and 3(b), which correspond to uniformly sampled data, might give the misleading impression that some mechanism is destroying forbidden patterns. However, both the decreasing trend in the chaotic regime and the constant curve in the periodic regime can be explained by considering the topological entropy $h_{\text {top }}(\mathbf{T})$ of the dynamics, where $\mathbf{T}$ denotes the discrete map that acts as the (numerical) approximation of the continuous system. Sampling at different frequencies leads to the collection of data corresponding to some integer multiple iterate of this map, $\mathbf{T}^{(\mathbf{n})}$. In the chaotic case, one may evaluate $h_{\text {top }}(\mathbf{T})$ of successive iterates $\mathbf{T}^{(\mathbf{n})}, \mathbf{T}^{(2 n)}, \ldots$ such that they match successive $\Delta t$ values. This yields decreasing values of the form $1-e^{m\left[n h_{\text {top }}(\mathbf{T})-\ln (m)+1\right]}$ due to the relation between the outgrowth rate of admissible patterns to $h_{\text {top }}(\mathbf{T})^{19,22}$ and explains the trend. In the periodic case, the topological entropy of the map is zero and thus the curve is expected to be constant.

This analysis is consistent with the results obtained from the 8-periodic Rössler (results omitted) and the hyperchaotic 4D Rössler (Fig. 4) time series. The curves are characterised by similar trends. All $m \leq 6$ curves eventually decay to zero once sampling is sufficiently sparse on average. The $m=7$ curve presumably decays to zero at $\Delta t \simeq 2$. The emergence of false admissible patterns responsible for the drop in $\mathcal{P}_{f}^{(m)}$ is evident even for small mean sampling intervals. The one difference in this case is that the estimator displays improved accuracy for very fine sampling resolutions. This is clear by the juxtaposition of the $m=7$ and $m=7(R)$ curves for small $\Delta t$ between Figs. 3(a), 3(b), and 4. The discrepancy in the $\mathcal{P}_{f}^{(m)}$ estimates computed from the hyperchaotic 4D Rössler is lower for all $\Delta t$.

Fig. 5 depicts the corresponding results for Pareto distributed sampling intervals with the scale parameter analogous to the interval size of a regular grid. Surprisingly,

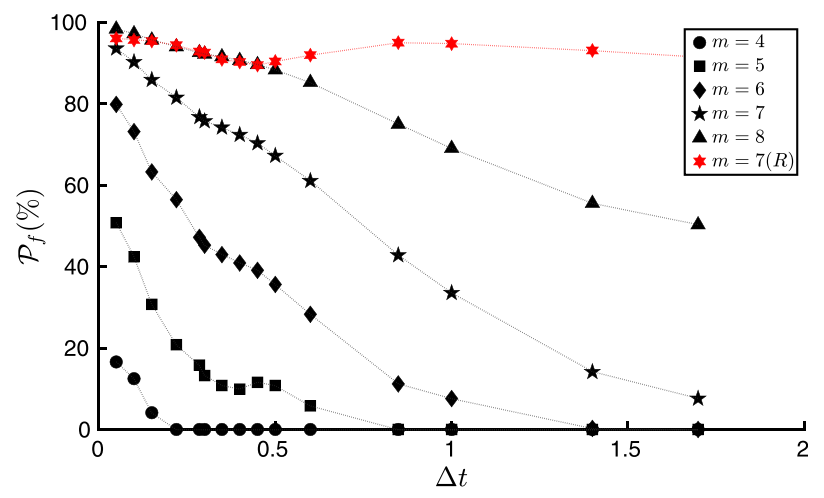

FIG. 4. Proportion of forbidden patterns in hyperchaotic 4D Rössler time series sampled by means of a Poisson process for various intensities with mean sampling interval $\Delta t$ and $4 \leq m \leq 8$. The $m=7$ curve for regularly sampled data of fixed interval $\Delta t$ is shown in red.
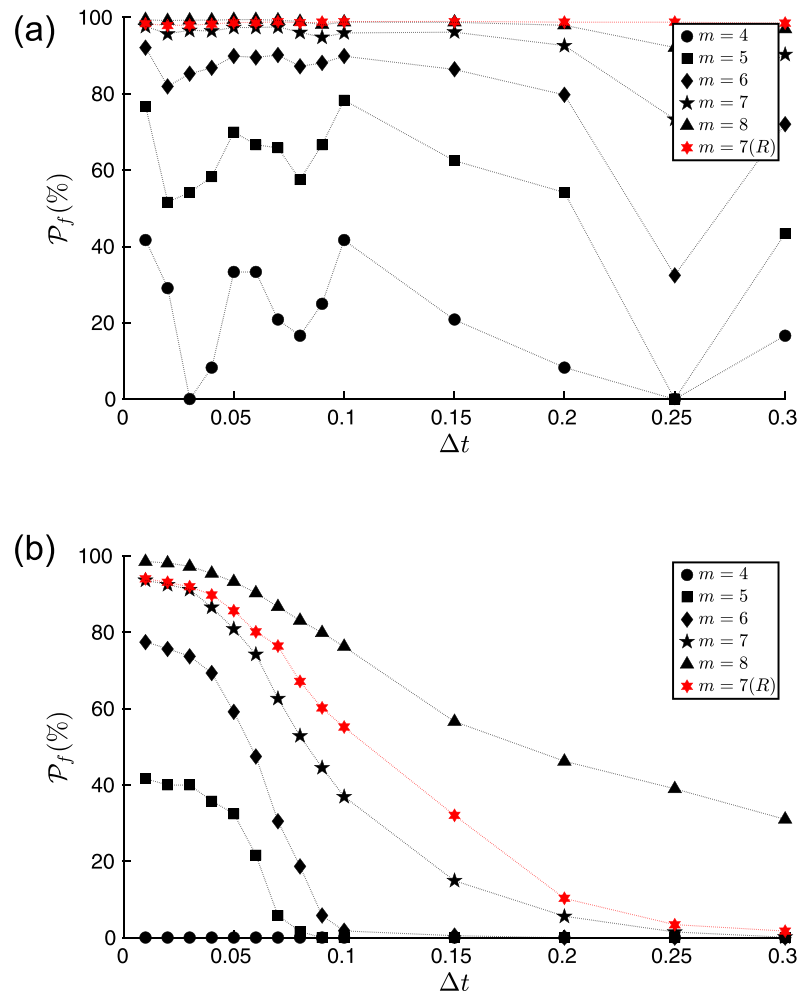

FIG. 5. Proportion of forbidden patterns in (a) periodic and (b) chaotic Lorenz time series with sampling intervals drawn from a Pareto distribution by varying the scale parameter $(=\Delta t)$ and $4 \leq m \leq 8$. The $m=7$ curve for regularly sampled data of the fixed interval $\Delta t$ is shown in red.

Pareto sampling with moderate sampling gaps is not detrimental for the estimation of the proportion of true forbidden patterns as long as $m$ is not too small, which is clear from the periodic case (Fig. 5(a)). When $m \geq 7$, the regularity of this dynamical regime is evidenced by the fact that $\mathcal{P}_{f}^{(m)}$ is close to 1 as expected. The results for the chaotic trajectory (Fig. 5(b)), in particular, the comparison between the $m=7$ cases, confirm that Pareto sampling has relatively mild effects on the estimator of forbidden patterns, without a major departure from the results obtained using uniformly sampled data.

A sensible conjecture is that as long as the sampling scheme contains enough "dense" areas, i.e., finely sampled, the existence of large sampling gaps does not alter the estimated proportion of forbidden patterns that much-as long as the mean sampling interval is not too large or the pattern length too small. To emphasize this point, Fig. 6 shows a comparison between regular, Poisson and Pareto sampling for $m=7$ and various $\Delta t$. For comparison, we also sampled from a $\Gamma(1, \Delta t)$ distribution, which corresponds to exponentially distributed sampling intervals of mean equal to $\Delta t^{-1}$. Pareto sampling is virtually indistinguishable from regular sampling if the resolution is sufficiently fine (here $\Delta t \leq 0.07$, $\sim 14$ points per unit time) in the periodic system. In the chaotic data set, this is only the case for $\Delta t \leq 0.04$, but the difference from regular sampling is never larger than $20 \%$, even for very coarse-grained sampling.

Exponentially distributed sampling intervals, either from a Poisson process or from a $\Gamma(1, \Delta t)$ distribution, lead to severe underestimates of the relative count of forbidden 

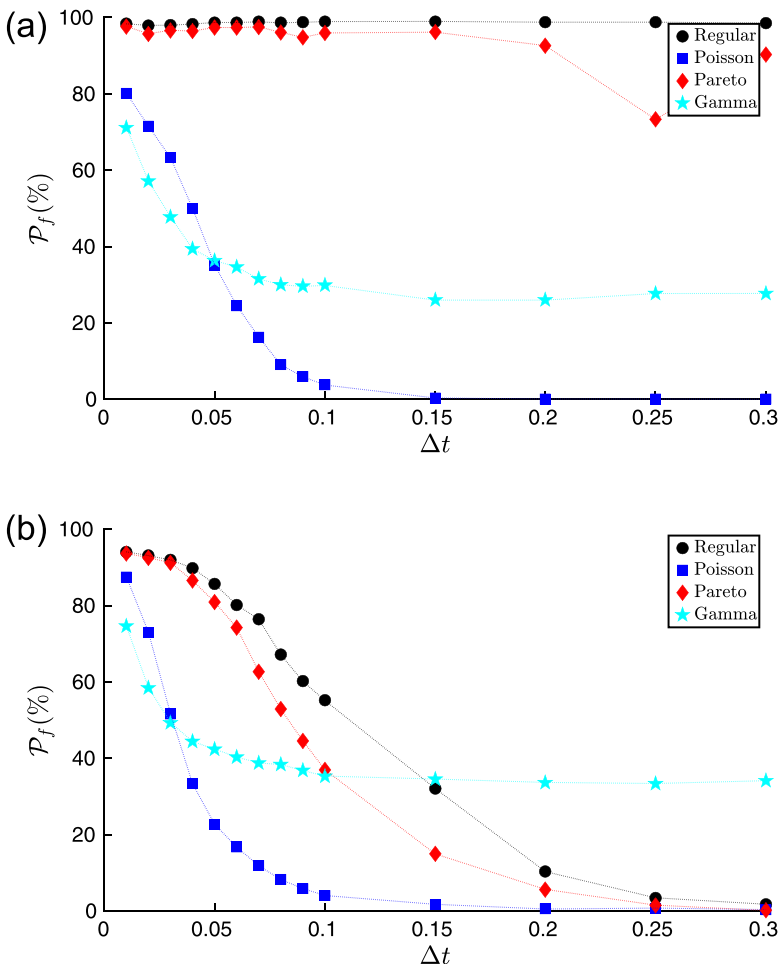

FIG. 6. Proportion of forbidden patterns in (a) periodic and (b) chaotic Lorenz time series $(m=7)$ sampled in four different ways. Regularly at fixed interval $\Delta t$, as a Poisson process of intensity $\Delta t$, and with sampling intervals drawn from $\operatorname{Pareto}(\Delta t, 10)$ and $\Gamma(1, \Delta t)$ distributions.

patterns. This is true even for small $\Delta t$. There is one key difference between these two schemes, however, as the $\Gamma$-distributed data were sampled at a mean sampling interval of $\Delta t^{-1}$. Therefore, the interpretation of the results of Fig. 6 in the case of this sampling scenario is different altogether. When $\Delta t$ is very small, consider 0.01 or 0.05 for instance, the corresponding mean sampling intervals are 100 and 20, respectively. The relatively high count of forbidden patterns here is owing to the fact that the time series has in fact been severely undersampled, there are insufficient data. As $\Delta t$ increases, the mean sampling interval reduces and more data points lead to more visible patterns, and hence a lower count of forbidden ones. This decrease will presumably continue and lead to a decay to zero until $\Delta t \simeq 10$. Afterwards, the mean sampling time will be less than 0.1 and $\mathcal{P}_{f}^{(m)}$ is expected to follow the results corresponding to the exponential curve (squares) in the reverse direction, thereby rendering meaningful estimates.

In summary, Pareto sampling within the parameter range outlined above does not significantly affect the relative count of forbidden patterns. Poisson sampling, in stark contrast, severely affects the estimated proportion of forbidden patterns and produces underestimates due to the presence of several false admissible patterns. However, the estimator is still highly robust if the mean sampling interval is sufficiently small. For chaotic systems, the time it takes for the autocorrelation function to cross zero may be used as a heuristic for a threshold.

The aforementioned observations lead to the question of whether one could potentially classify irregular sampling schemes based on their effects on the reliability of the forbidden patterns' estimator in detecting determinism. To this end, we consider $\Gamma$-sampling by varying the skewness for a fixed mean sampling interval $\Delta t$. The different probability density functions corresponding to various degrees of skewness (different colours) are shown in Fig. 7(a) for a portion of the domain we have elected to use, $\Delta t \in[0,0.3]$, along with the relevant logarithmic-scale plot on the full domain (Fig. 7(b)) for large values of the skewness (exponential and power-law behaviour). The associated complementary cumulative distribution functions (cdf) are shown in Fig. 7(c).

Fig. 8 shows the relative count of forbidden patterns for various skewness levels. When $\operatorname{Skew}\left(t_{k}\right)$ is very small, the shape parameter is large and the distribution of sampling intervals is essentially normal, centered around 0.05 . The lowest value we chose is $\operatorname{Skew}\left(t_{k}\right)=0.1$, which yields a
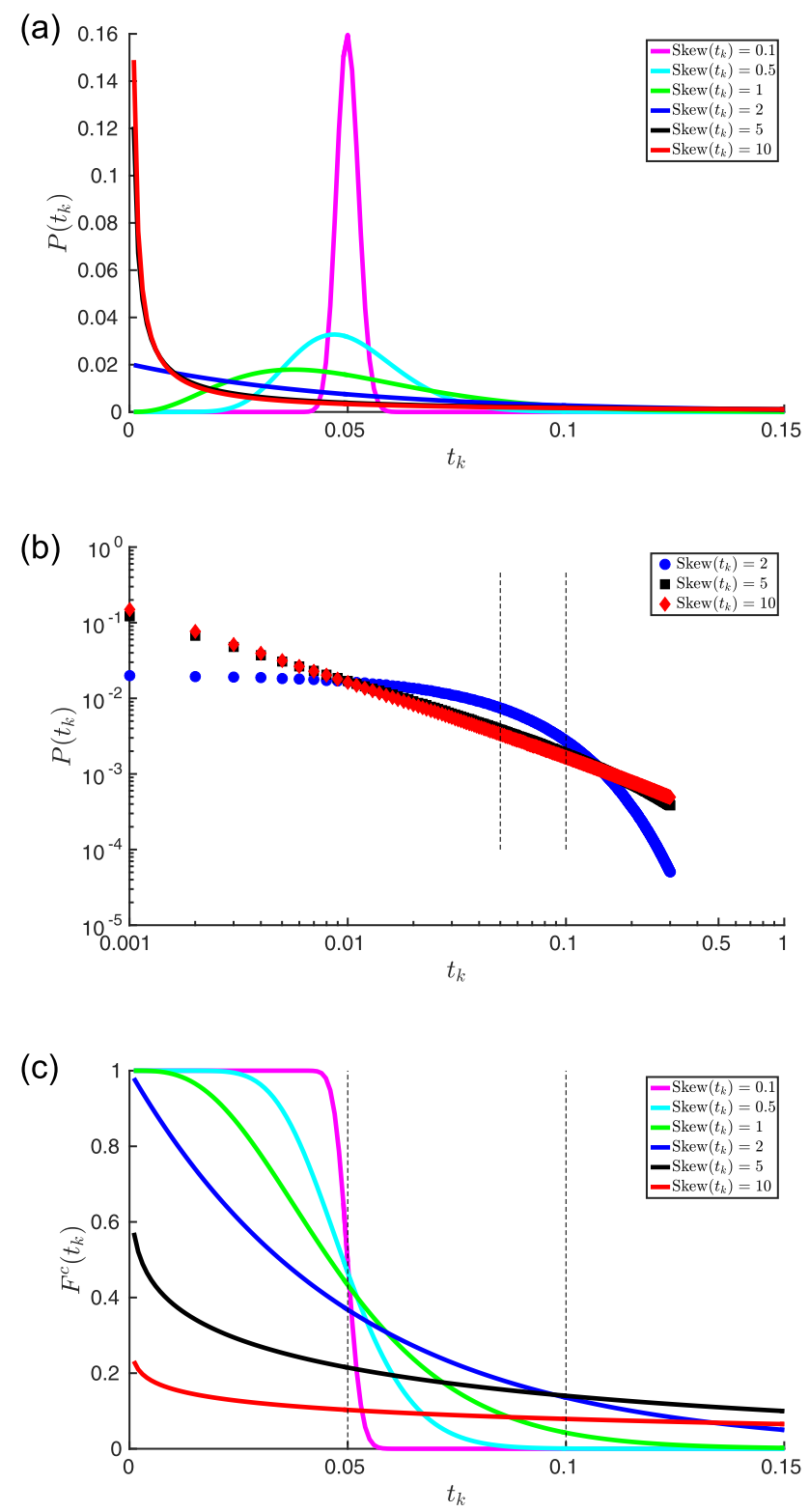

FIG. 7. Probability density function (top), corresponding logarithmic-scale plot (middle) and complementary cumulative distribution function $F^{c}\left(t_{k}\right)=\operatorname{Pr}\left[\mathcal{T}_{k} \geq t_{k}\right]$ (bottom) for $\Gamma$-distributed sampling intervals of various degrees of skewness and mean $\Delta t$. 
(a)

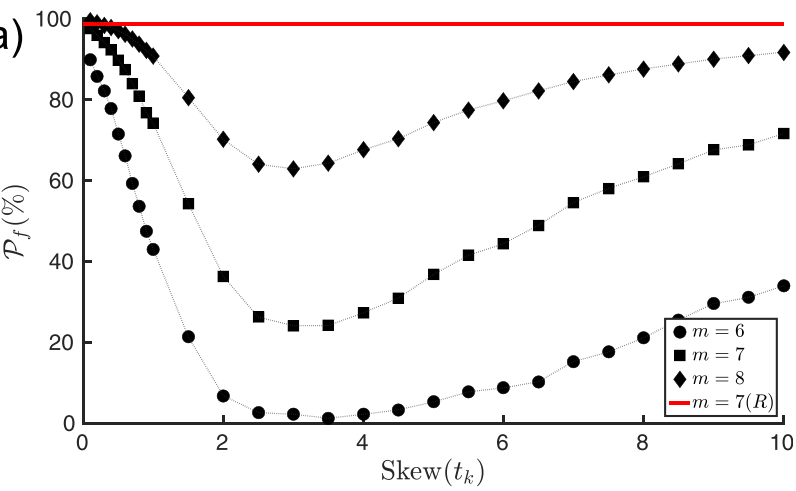

(b)

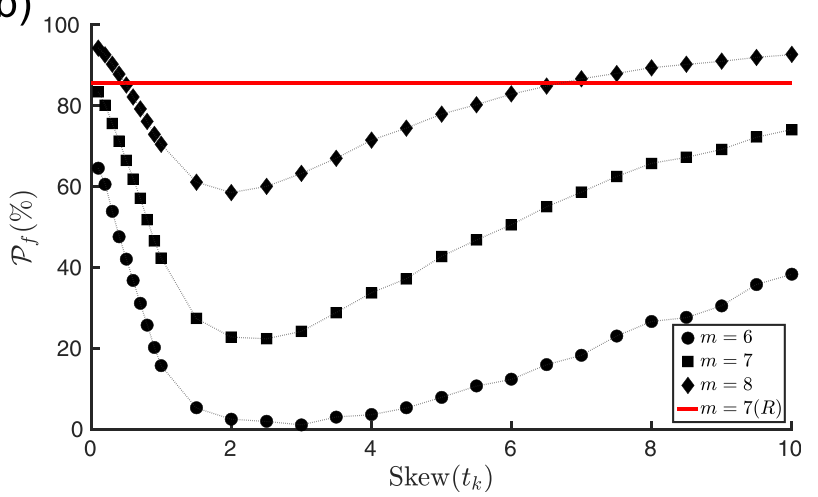

FIG. 8. Proportion of forbidden patterns in (a) periodic and (b) chaotic Lorenz time series obtained using $\Gamma$-distributed sampling intervals with mean $\Delta t$ as a function of the skewness for $6 \leq m \leq 8$. The corresponding count for regularly sampled data of fixed interval $\Delta t$ and $m=7$ is shown in red.

variance of $1.25 \times 10^{-4}$. The sampling scheme in this case is very similar to a regular grid with small timing jitter. The top panel of Fig. 8 confirms that $\mathcal{P}_{f}^{(m)}$ is close to $100 \%$ for all $m$, as expected for a periodic regime. In fact for $m=7$, the value almost coincides with the one obtained from regularly sampled data. The situation is analogous in the chaotic case as the proximity to the regular grid reference line indicates. Insufficiency of data with respect to 8 ! causes an overestimate in the case of $m=8$. The discrepancy between the estimates for the regularly and the irregularly sampled data when $m=7$ and the skewness is small is of the order of $1 \%$.

Once we start increasing the skewness, $\mathcal{P}_{f}^{(m)}$ drops very fast reaching a minimum in the region $2 \leq \operatorname{Skew}\left(t_{k}\right) \leq 4$. Note that $\operatorname{Skew}\left(t_{k}\right)=2$ corresponds to sampling by means of a Poisson process with exponentially distributed sampling intervals. This result confirms the conclusions reached previously; this scheme seems to be one of the worst for accurately estimating the proportion of true forbidden patterns admitted by a time series.

Once the skewness of the sampling distribution increases even further (intuitively making it more asymmetric), its density function approaches a power law (see middle panel of Fig. 7 above), rendering larger chronological gaps in the sampling scheme more frequent. In contrast to typical "fat-tailed" distributions like Weibull's or Pareto's with a very small tail index, however, the exponential decay term ensures that densely sampled areas also occur, and in fact much more frequently.
This phenomenon can be better explained by looking at Fig. 7. Keeping in mind that the mean sampling interval is the same for all distributions, we examine the heterogeneity of the sampling distribution through the lens of the complementary cdf $P\left[t_{k} \geq t^{*}\right]$ (Fig. 7(c)). In particular, we observe the frequency of extreme values in a statistical sense. How many densely sampled areas $\left(t_{k} \rightarrow 0\right)$ exist? How many large chronological gaps $\left(t_{k} \rightarrow \infty\right)$ ? Fine-resolution regions consist of data points whereby temporal neighbours are highly correlated (if generated by deterministic rules) and lead to increased robustness in terms of the forbidden patterns' estimator. Large chronological gaps lead to measuring successive values that are not correlated and the consequent introduction of false admissible patterns. However, the length of these large gaps is not so important since its presence can give rise to at most $m-1$ false admissible patterns. It is rather the frequency of larger gaps than a certain threshold that is of utmost significance. Since the autocorrelation function crosses zero at approximately $t=0.05$ for our chaotic Lorenz data, we propose this value as a threshold. By looking at Fig. 7(c) and $P\left[t_{k} \geq 0.05\right]$ specifically, we observe that the frequency of larger $\geq 0.05$ sampling intervals is much higher for the normal distributions (S-shaped, magenta, cyan, and green in colour) and the exponential (blue) than the power-law distributions (black and red curves). However, intermediate-sized sampling intervals are produced at a significantly higher rate by normally distributed sampling schemes and so the complementary cumulative distribution drops very rapidly. In fact, exponentially distributed data exhibit the highest probability of intervals in the range $0.05 \leq t_{k} \leq 0.1$. Additionally, it is evident from all panels of Fig. 7 that densely sampled areas are more frequent if the distribution is more asymmetric and exhibits powerlaw behaviour. At the same time, Fig. 7(b) shows that such sampling distributions are also characterised by more largesized sampling intervals $\left(t_{k} \geq 0.2\right)$ in comparison to more symmetric ones. Despite this, their overall probability is orders of magnitude lower and outbalanced by the much more frequent densely sampled areas. Consequently, we deduce that the proportion of true forbidden patterns may be estimated accurately when sampling deterministic systems in a non-uniform manner, even in the presence of very large sampling intervals, as long as there is a sufficiently high number of densely sampled "fine-resolution" areas.

Fig. 9 depicts the corresponding results from experiments conducted on 8-periodic Rössler and hyperchaotic 4D Rössler time series. Results are in accordance with our analysis on the Lorenz data. The $\mathcal{P}_{f}^{(m)}$ estimator produces similar values to those corresponding to uniformly sampled data if the level of skewness of the sampling distribution is very small. In addition, as the top panel of Fig. 9 illustrates, $\mathcal{P}_{f}^{(m)}$ produces stable values for a wider spectrum of skewness levels in comparison to the chaotic or hyperchaotic cases. This stability is also evident in the periodic Lorenz case (Fig. 8(a)), albeit at a somewhat lesser degree as the Lorenz time series is generated by a slightly more complex limit cycle mechanism in phase space. This robustness in periodic data can be explained by the fact that $\mathcal{P}_{f}^{(m)}$ can capture a deterministic component in strongly periodic or quasi-periodic 
(a)

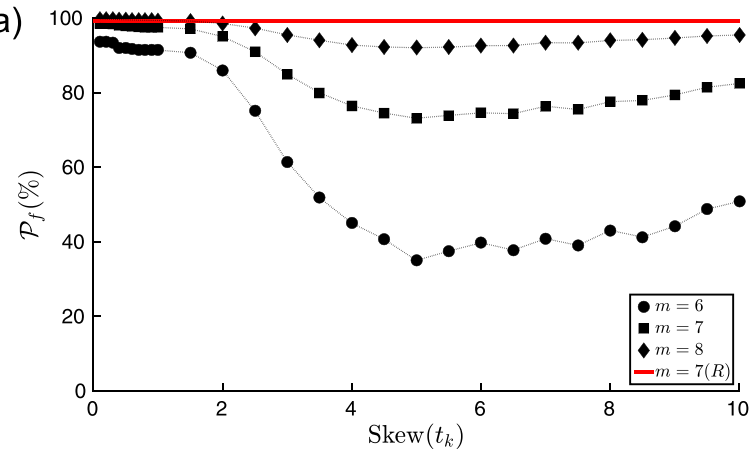

(b)

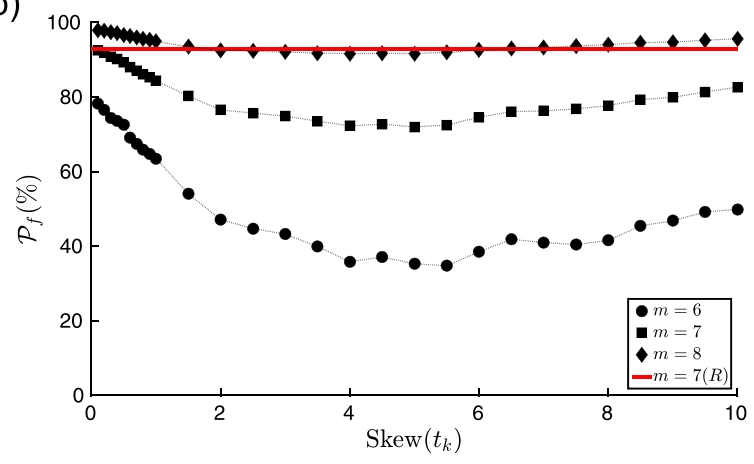

FIG. 9. Proportion of forbidden patterns in (a) 8-periodic Rössler and (b) hyperchaotic 4D Rössler time series obtained using $\Gamma$-distributed sampling intervals with mean $\Delta t$ as a function of the skewness for $6 \leq m \leq 8$. The corresponding count for regularly sampled data of fixed interval $\Delta t$ and $m=7$ is shown in red.

data even at very coarse sampling resolutions (although at a multiple of the underlying period). In periodic time series, the regularity of sampling points is more significant than the average sampling density, which is in agreement with our previous findings. ${ }^{21}$ This phenomenon may also be observed by comparing the chaotic Lorenz results (Fig. 8(b)) with the hyperchaotic Rössler results (Fig. 9(b)). The latter is a more complex system that, however, exhibits a strong quasiperiodic character in the phase-space projection onto the $x$-coordinate (also manifested in the associated autocorrelation function). Therefore, the $\mathcal{P}_{f}^{(m)}$ estimator produces values closer to the regular sampling benchmark even for less symmetric distributions in the region $0 \leq \operatorname{Skew}\left(\mathrm{t}_{\mathrm{k}}\right) \leq 1$ since fewer false admissible patterns emerge.

Furthermore, at high skewness levels we observe high $\mathcal{P}_{f}^{(m)}$ estimates, which slowly approach the regular sampling benchmark. Note that the estimates are more accurate in the hyperchaotic data. In this parameter region, there is a simultaneous presence of large chronological gaps and densely sampled regions as discussed above. In periodic data whereby the regularity of sampling is of primary concern, this leads to more false admissible patterns. In chaotic or hyperchaotic data whereby the average sampling density in comparison to the decorrelation/Lyapunov time of the system is of utmost significance, $\mathcal{P}_{f}^{(m)}$ estimates are more stable. This can also be seen in the Lorenz case (see $m=7$ curves in Figs. 8(a) and 8(b)). Finally, if the level of skewness is intermediate, $\mathcal{P}_{f}^{(m)}$ produces relatively poor estimates, just as in the Lorenz case. A notable difference, however, is that $\mathcal{P}_{f}^{(m)}$ values are generally higher and more stable for these systems, in contrast to the Lorenz results. Our hypothesis is that stronger periodic behaviour minimises the potential for false admissible patterns and thus we observe slightly higher $\mathcal{P}_{f}^{(m)}$ values by virtue of the nature of these systems.

\section{CONCLUSIONS}

The numerical experiments conducted herein allowed us to examine the robustness of the estimator of the relative count of forbidden patterns with respect to various irregularly sampled time series. We considered sampling intervals from exponential, Pareto and Gamma distributions and established the conditions under which the count of forbidden patterns can be a reliable criterion for detecting determinism. Our findings indicate that irregular sampling plays an important role in the resulting estimate and certain sampling distributions are preferable to others in this sense. While an experimentalist does not have the freedom of choosing a particular sampling scheme in general, given knowledge of the sampling distribution one can make a heuristic evaluation about the reliability of this measure as a criterion for detecting determinism in this data set.

As our previous investigation ${ }^{21}$ showed, the pattern length parameter needs to be set as large as the length of the data set permits. This is in order to minimise the existence of both false admissible and false forbidden patterns, thereby eliminating effects of uncorrelated time series points and undersampling, respectively. Additionally, for periodic systems the regularity of the sampling scheme is more important than the mean sampling interval, as periodicity is still captured well from a forbidden patterns perspective even at periods greater than the original. On the contrary, in chaotic systems it is more important to ensure a mean sampling interval within the range where correlation is still observed in the autocorrelation function.

Sampling by means of a Poisson point process can lead to gross underestimation of the proportion of true forbidden patterns-unless the mean sampling interval is very low. This is because of the creation of many false admissible patterns when many larger-sized intervals than the critical correlation time are counted. Such patterns should not occur but are rather a consequence of this particular manner of sampling, which leads to many decorrelated pairs of successive time series points. Therefore, forbidden patterns' estimates can potentially be very inaccurate when the sampling distribution is exponential. Necessary conditions include a sufficiently large pattern length, a long enough data set relative to $m$ !, and a smaller mean sampling time than a typical decorrelation time, otherwise distinction between determinism and randomness may become unreliable.

Our most significant finding is that estimates of forbidden patterns do not suffer significantly in the presence of moderate or even large chronological gaps if certain conditions are met. This type of sampling is common in geoscientific measurements and, therefore, forbidden patterns will likely be a reliable tool in detecting determinism in such data sets. To simulate this sampling situation, we considered Pareto and $\Gamma$-sampling distributions with various degrees of skewness. Very low or high skewness levels produce 
distributions, which lead to reliable forbidden patterns estimates as long as the requirements of large pattern length and small mean sampling interval are fulfilled. At high skewness levels, we observed a reliability of the estimate, which was more unexpected. The primary condition here is to ensure a sufficiently high frequency of densely sampled areas (i.e., small sampling intervals), considerably higher than the number of large chronological gaps.

Consequently, we propose that the first moment and the degree of asymmetry of an empirical sampling distribution may be used as heuristic indicators in order to assess the reliability of the estimate of the count of forbidden patterns. Thereby, one can further assess the potential for detection of determinism using this measure. In this regard, either very high symmetry-additionally characterised by very thin tails and small variance — or complete asymmetry with moderate to fat tails is strongly preferred. Such sampling regimes minimise the recording of both false admissible and false forbidden patterns.

\section{ACKNOWLEDGMENTS}

M.S. is supported by the Australian Research Council Discovery Project No. DP 140100203.

${ }^{1}$ H. Bungum, T. Risbo, and E. Hjortenberg, J. Geophys. Res. 82, 5365, doi:10.1029/JB082i033p05365 (1977).
${ }^{2}$ M. H. Trauth, J. C. Larrasoana, and M. Mudelsee, Quat. Sci. Rev. 28, 399 (2009).

${ }^{3}$ K. Rehfeld, N. Marwan, J. Heitzig, and J. Kurths, Nonlinear Processes Geophys. 18, 389 (2011).

${ }^{4}$ F. H. McRobie, T. Stemler, and K.-H. Wyrwoll, Quat. Sci. Rev. 121, 120 (2015).

${ }^{5}$ P. Vanicek, Astrophys. Space Sci. 12, 10 (1971).

${ }^{6}$ N. R. Lomb, Astrophys. Space Sci. 39, 447 (1976).

${ }^{7}$ J. D. Scargle, Astrophys. J. 263, 835 (1982).

${ }^{8}$ K. Rehfeld and J. Kurths, Clim. Past 10, 107 (2014).

${ }^{9}$ S. Suzuki, Y. Hirata, and K. Aihara, Int. J. Bifurcation Chaos 20, 3699 (2010).

${ }^{10}$ I. Ozken, D. Eroglu, T. Stemler, N. Marwan, G. B. Bagci, and J. Kurths, Phys. Rev. E 91, 062911 (2015).

${ }^{11}$ D. Eroglu, F. McRobie, I. Ozken, T. Stemler, K.-H. Wyrwoll, S. F. M. Breitenbach, N. Marwan, and J. Kurths, Nat. Commun. 7, 12929 (2016).

${ }^{12}$ C. Bandt and B. Pompe, Phys. Rev. Lett. 88, 174102 (2002).

${ }^{13}$ Y. Cao, W. Tung, J. B. Gao, V. A. Protopopescu, and L. M. Hively, Phys. Rev. E 70, 046217 (2004).

${ }^{14}$ A. Groth, Phys. Rev. E 72, 046220 (2005).

${ }^{15}$ K. Keller and H. Lauffer, Int. J. Bifurcation Chaos 13, 2657 (2003).

${ }^{16}$ U. Parlitz, S. Berg, S. Luther, A. Schirdewan, J. Kurths, and N. Wessel, Comput. Biol. Med. 42, 319 (2012).

${ }^{17}$ J. M. Amigó, L. Kocarev, and J. Szczepanski, Phys. Lett. A 355, 27 (2006).

${ }^{18}$ J. M. Amigó, S. Zambrano, and M. A. F. Sanjuán, EPL (Europhys. Lett.) 83, 60005 (2008).

${ }^{19}$ C. Bandt, G. Keller, and B. Pompe, Nonlinearity 15, 1595 (2002).

${ }^{20}$ C. W. Kulp, J. M. Chobot, B. J. Niskala, and C. J. Needhammer, Chaos 26, 023107 (2016).

${ }^{21}$ M. McCullough, K. Sakellariou, T. Stemler, and M. Small, Chaos 26, 123103 (2016).

${ }^{22}$ J. M. Amigó, S. Zambrano, and M. A. F. Sanjuán, Europhys. Lett. 79, 50001 (2007). 